\title{
DNA Methyltransferase 3 (MET3) is regulated by Polycomb group complex during Arabidopsis endosperm development
}

\author{
Louis Tirot $^{1} \cdot$ Diane M. V. Bonnet ${ }^{1} \cdot$ Pauline E. Jullien $^{1}$ (D)
}

Received: 28 July 2021 / Accepted: 14 December 2021 / Published online: 28 January 2022

(c) The Author(s) 2021

\begin{abstract}
Complex epigenetic changes occur during plant reproduction. These regulations ensure the proper transmission of epigenetic information as well as allowing for zygotic totipotency. In Arabidopsis, the main DNA methyltransferase is called MET1 and is responsible for methylating cytosine in the CG context. The Arabidopsis genome encodes for three additional reproductionspecific homologs of MET1, namely MET2a, MET2b and MET3. In this paper, we show that the DNA methyltransferase MET3 is expressed in the seed endosperm and its expression is later restricted to the chalazal endosperm. MET3 is biallelically expressed in the endosperm but displays a paternal expression bias. We found that MET3 expression is regulated by the Polycomb complex proteins FIE and MSI1. Seed development is not impaired in met 3 mutant, and we could not observe significant transcriptional changes in met 3 mutant. MET3 might regulates gene expression in a Polycomb mutant background suggesting a further complexification of the interplay between H3K27me3 and DNA methylation in the seed endosperm.
\end{abstract}

Key message The DNA METHYLTRANSFERASE MET3 is controlled by Polycomb group complex during endosperm development.

Keywords DNA methylation $\cdot$ Arabidopsis $\cdot$ Endosperm $\cdot$ Polycomb $\cdot$ MET3

\section{Introduction}

Sexual reproduction in Angiosperm is initiated by a double fertilization event. Fertilization of the haploid egg cell by one of the sperm cells gives rise to the diploid embryo, whereas fertilization of the homodiploid central cell gives rise to the triploid endosperm (Berger 2003; Costa et al. 2004). The endosperm represents a nourishing tissue supporting embryo growth and is therefore key for proper seed development. Cell divisions in the endosperm are initiated very rapidly following fertilization. These divisions are initially occurring without cellularization and form a syncytium that will later cellularize (Brown et al. 1999, 2003; Boisnard-Lorig et al. 2001). An additional peculiarity of the endosperm, beyond its triploid syncytial nature, is being the seat of interesting epigenetic phenomena and complex epigenetic regulation.

Communicated by Claudia Köhler.

Pauline E. Jullien

pauline.jullien@ips.unibe.ch

1 Institute of Plant Sciences, University of Bern, Bern, Switzerland
Endosperm development and cellularization are indeed regulated by the FIS Polycomb group complex known to mediate Histone H3 Lysine 27 tri-methylation, a key silencing epigenetic mark. Some of the key members of the FIS Polycomb group complex (FIS-PcG) are MEA, FIS2, FIE, and MSI1 (Chaudhury et al. 1997; Luo et al. 1999; Kiyosue et al. 1999; Yadegari et al. 2000; Köhler et al. 2003; Guitton et al. 2004). In mutants affecting those genes, the endosperm fails to cellularize, resulting in an arrest of embryo development and eventually seed abortion. Additionally, several genes were found to be imprinted, i.e., only one parental allele is expressed, whereas the other is epigenetically silent, in the endosperm. This is the case, for example, of genes such as FIS2, FWA, MEA or PHE1 (Kinoshita et al. 1999, 2004; Luo et al. 2000; Köhler et al. 2005; Jullien et al. 2006). The silencing of those endosperm imprinted genes relies principally on two epigenetic mechanisms: either regulation by the FIS-PcG itself like for $M E A$ or $P H E 1$ or silencing by DNA methylation like for FWA and FIS2 (Jullien and Berger 2009; Gehring 2013; Batista and Köhler 2020). Another epigenetic singularity of the endosperm is of being relatively hypomethylated compared to other plant tissues (Gehring et al. 2009; Hsieh et al. 2009). This hypomethylation is due in part to the activity of a DNA glycosylase called 
DEMETER (Choi et al. 2002; Gehring et al. 2006; Hsieh et al. 2009) and likely also to the low expression of canonical actors of the DNA methylation pathway (Jullien et al. 2012).

DNA methylation is a key epigenetic mark regulating gene expression and protecting genome integrity by repressing transposons. In plant genomes, DNA methylation is found in three cytosine contexts: CG, CHG and CHH (where $\mathrm{H}$ is any base except $\mathrm{C}$ ). Methylation on these different contexts relies on specific DNA methyltransferases. DNA methylation on CG sites relies on maintenance DNA METHYLTRANSFERASE (MET) where the main ubiquitous enzyme is MET1. DNA methylation on CHG sites relies on CHROMOMETHYLASE3 (CMT3) and an interplay with histone methylation (Lindroth et al. 2001). DNA methylation on $\mathrm{CHH}$ site, due to its non-symmetrical nature, relies on the constant de novo methylation pathway involving small RNA molecules as well as DOMAIN REARANGED METHYLTRANSFERASE2 (DRM2) (Cao and Jacobsen 2002). In centromeric sequences, $\mathrm{CHH}$ methylation also relies on CHROMOMETHYLASE2 (CMT2) (Stroud et al. 2013).

Although we know a lot about the main actors of this pathway, the Arabidopsis's genome encodes multiple copies of DNA methyltransferase genes (4 METs, 3 CMTs and $3 D R M s$ ) some of which might have a more complex or similar function in discreet cell types. For example, CMT1 (the third CHROMOMETHYLASE encoded by the Arabidopsis genome) is principally detected in reproductive tissue (Henikoff and Comai 1998; Klepikova et al. 2016), and the reconstitution of a full-length transcript relies on the splicing out of a transposable element situated in its 13th exon (Yadav et al. 2018). The DOMAIN REARANGED METHYLTRANSFERASE1 (DRM1) seems to also solely play a role in reproductive tissue, where a redundancy between $D R M 1$ and DRM2 was observed in the early embryo (Jullien et al. 2012). Similarly, data concerning the potential function of non-canonical METs are scarce. MET2 $a$ and MET2 $b$ are detected in the central cell, but their function is unknown (Jullien et al. 2012). Nonetheless, correlative evidence suggest MET2 $a$ might be important to regulate transposon reactivation in wild Arabidopsis accessions (Quadrana et al. 2016) and involved in fungal response (Salvador-Guirao et al. 2018).

As mentioned, little is known about the DNA methyltransferase MET3. MET3 is also named MATERNAL EFFECT EMBRYO ARREST 57 (MEE57) as a transposon insertion associated with the MET3 locus led to an arrest in endosperm development (Pagnussat et al. 2005). MET3 is also reported to be the sole MET expressed in the endosperm (Jullien et al. 2012). Here, we show that MET3 is specifically expressed in the endosperm in a biallelic fashion with a paternal bias. MET3 expression is controlled by the FIS-PcG complex. Despite the initial report of a seed arrest phenotype in the mee 57 line, we did not see any seed developmental phenotype in two independent met 3 mutant alleles. Additionally, we could not see major changes in the seed transcriptome of met 3 mutant. Nevertheless, we could see a minor effect on the seed transcriptome in a fie mutant background suggesting that MET3 might interplay with PcG gene regulation in the developing endosperm.

\section{Materials and methods}

\section{Plant materials, growth conditions and genotyping.}

The wild-types Col-0 and Gr-1, the MET3 mutant lines met3-3 (GABI404F04), met3-4 (GABI659H03) and PRC2 mutant lines fie-362 (GABI_362D08) (Bouyer et al. 2011) and msil (SAIL_429_B08) were provided by the Nottingham Arabidopsis Stock Center (NASC). pMET3:H2B-RFP line was previously described (Jullien et al. 2012). After sowing, plants were stratified in the dark at $4{ }^{\circ} \mathrm{C}$ between 2 and 4 days. Plants were germinated and grown in growing chambers under long-day conditions $\left(16 \mathrm{~h}\right.$ light $22^{\circ} \mathrm{C} / 8 \mathrm{~h}$ dark $18^{\circ} \mathrm{C}$ ). For the transmission analysis (Fig. S5a), plants were grown on Murashige and Skoog (MS1/2) media agarose plate in long-day conditions for 12 days before genotyping. Primers for genotyping are listed in Table S1.

\section{Microscopy and phenotype observation}

DIC seed phenotype and GUS observations were done using a Leica DM2000 as previously described (Jullien et al. 2006). For seed development observation and counting (Fig. 4), plants were synchronized by removing all open flowers from the inflorescences. After 6 days, the two first siliques situated above the previously removed flowers were picked for each inflorescence. We refer to this stage as 6 days after synchronization (6 DAS). A side-by-side developmental comparison of DAP and DAS can be found in Fig. S6. Siliques were dissected, cleared using chloride hydrate solution and mounted on a slide for observation. pMET3:H2BtdTomato and pMET3:H2B-RFP reporter lines were imaged using a laser scanning confocal microscope (Leica SP5). For tdTomato reporters, samples were exited with a laser diode at $561 \mathrm{~nm}$ and reporter signal was captured by a Hybrid detector set between 600 and $660 \mathrm{~nm}$, while autofluorescence was captured by a PMT detector set between 667 and $800 \mathrm{~nm}$. For RFP reporters, samples were also exited with a laser diode at $561 \mathrm{~nm}$ and RFP signal was captured by a Hybrid detector set between 570 and $640 \mathrm{~nm}$ while autofluorescence was captured by a PMT detector set between 691 and $785 \mathrm{~nm}$. In both cases, $561 \mathrm{~nm}$ laser diode power was set between 50 and $80 \%$. When necessary, brightness and contrast were uniformly modified by using ImageJ. 


\section{Cloning and transformation}

pMET3:H2B-tdTomato and pMET3:H2B-GUS were generated using the Gateway Cloning System (Invitrogen). The destination vector is $\mathrm{pH} 7 \mathrm{~m} 34 \mathrm{GW}$ (Karimi et al. 2005) for pMET3:H2B-tdTomato and pAlli-R4R3-Tnos (Bensmihen et al. 2004) for pMET3:H2B-GUS. All PCR fragments were amplified by PCR using the Phusion High-Fidelity DNA Polymerase (Thermo). Primer sequences used for cloning can be found in Table S1. All plasmids were transformed into wild-type Columbia-0 plants the by floral dipping method (Clough and Bent 1998). At least ten transgenic lines were analyzed per construct, which showed a consistent fluorescence expression pattern. An Illustration of the constructs can be found in Fig. S2a.

\section{RNA extraction, qPCR \& RT-PCR}

Total RNAs were extracted using RNeasy Plant Minikit (Qiagen). All samples were treated with DNase I (ThermoScientific) at $37^{\circ} \mathrm{C}$ for $30 \mathrm{~min}$. DNAse I was subsequently inactivated by the addition of EDTA and heat treatment $\left(65{ }^{\circ} \mathrm{C}\right.$ for $\left.10 \mathrm{~min}\right)$. First-strand cDNAs were synthesized using between 500 and $1000 \mathrm{ng}$ of DNase treated total RNAs as a template. The RT reaction was performed using either Maxima First Strand cDNA Synthesis Kit (Figs. 1a, 2a) (ThermoScientific), containing both oligo-dT and random hexamer primers, or RevertAid RT Reverse Transcription Kit (Fig. 3e, Fig. S4b) (ThermoScientific), containing only oligo-dT primers. The qPCR reactions were performed with a QuantStudio 5 thermocycler (ThermoScientific) using SYBR green (KAPA SYBR FAST qPCR Master Mix or ORA qPCR Green ROX H Mix). The qPCR mix was prepared according to the manufacturer's protocol. An RNA equivalent of $25 \mathrm{ng}$ of cDNA templates was used for each reaction. The qPCR program was as follow: $95^{\circ} \mathrm{C}$ for $3 \mathrm{~min}$ followed by 45 cycles of $95^{\circ} \mathrm{C}$ for $5 \mathrm{~s}$ and $60{ }^{\circ} \mathrm{C}$ for $30 \mathrm{~s}$. ACTIN2 (AT3G18780) expression was used to normalize the transcript level in each sample. For each condition, RNA abundance of target genes was calculated from the average of three independent biological replicates with three qPCR technical replicates. Real-time PCR primers used in this study are listed in Table S1. For the allele-specific RT-PCR (Fig. 2a), cDNAs were amplified for 22 cycles for ACT2 primers and 35 cycles for MET3 specific primers. Half of the MET3 PCR product was digested for $1 \mathrm{~h} 30$ at $37^{\circ} \mathrm{C}$ with XbaI restriction enzyme (Sigma-Aldrich). ACT2 amplification was used as a control.
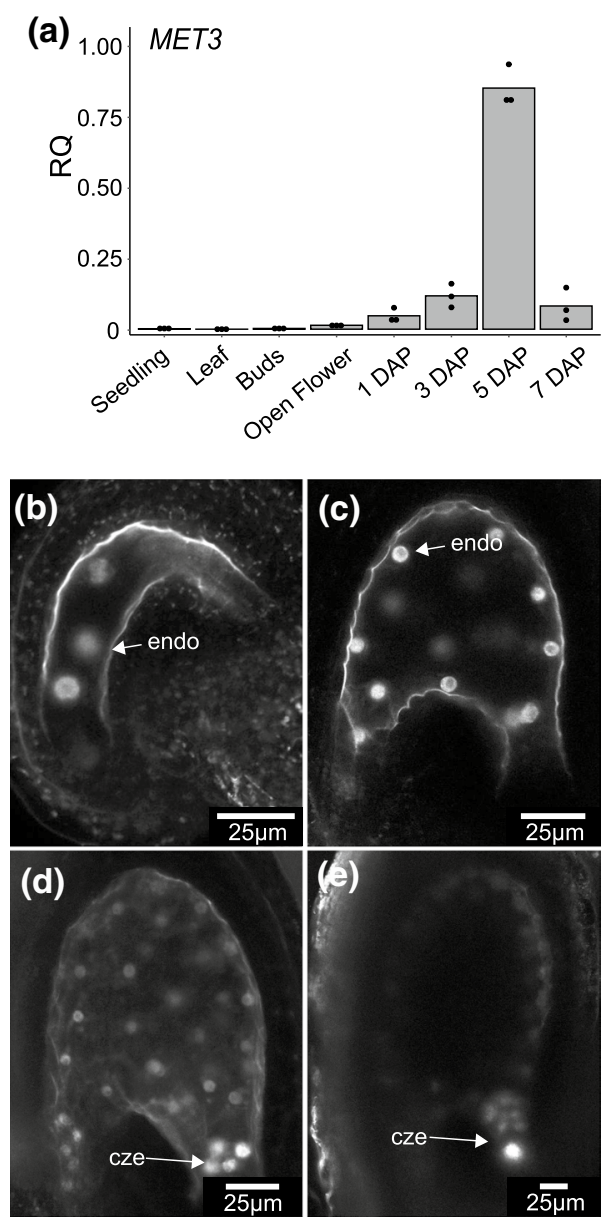

Fig. 1 MET3 expression pattern a MET3 expression measured by RT-qPCR. DAP = Day After Pollination. The histogram displays the mean, and each dot represents a biological replicate. ACT2 is used as a normalizer. $\mathrm{RQ}=$ Relative Quantification (b-e) Single-plan Confocal images representing the expression of pMET3:H2B-tdTomato construct in Arabidopsis 1 Day After Synchronization (DAS) seed b, 2 DAS seed c, 3 DAS d and 5 DAS e. cze $=$ chalazal endosperm, endo $=$ endosperm nuclei. Scale bars represent $25 \mu \mathrm{m}$

\section{RNA sequencing and Bioinformatics}

Total RNAs were extracted and DNAseI treated as previously mentioned. mRNA libraries were prepared and sequenced by Novogene (https://en.novogene.com/). Bioinformatic analyses were performed on the Galaxy web platform (https://usegalaxy.org) (Afgan et al. 2018). Our Galaxy workflow including the exact parameters and tool versions used can be downloaded on https://usegalaxy. $\mathrm{org} / \mathrm{u} / \mathrm{pej} / \mathrm{w} /$ pejrnaseq and be freely reused. Briefly, Pairedend raw mRNA sequencing reads were controlled using FastQC (Galaxy version 0.72) and trimmed using Trimmomatic (Galaxy version 0.36.6) (Andrews 2010; Bolger et al. 2014). Clean reads were aligned to the Arabidopsis thaliana TAIR 10 genome assembly using HISAT2 (Galaxy version 2.1.0+ galaxy4) (Kim et al. 2015). Aligned sequencing reads 

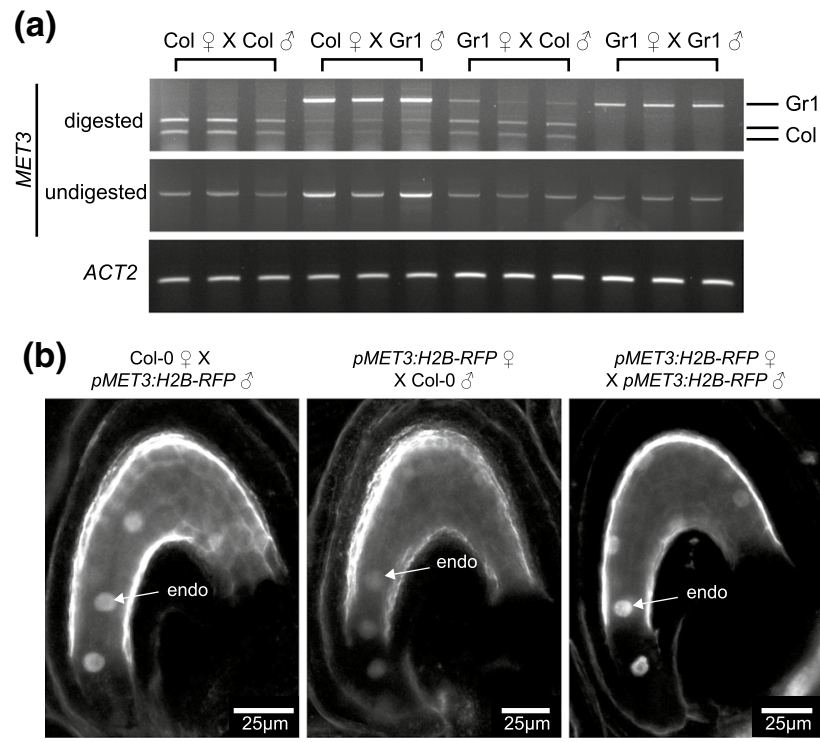

Fig. 2 MET3 is biallelically expressed with a paternal bias a Allele specific RT-PCR of MET3 parental expression in 5DAP silique samples. The XbaI restriction enzyme digests the Col-0 MET3 transcript but not the Gr-1 transcript. ACT2 is used as loading control. b Singleplan confocal images of pMET3:H2B-RFP parental expression in the endosperm of 1 DAP seeds. endo=endosperm nuclei. Scale bars represent $25 \mu \mathrm{m}$. $\delta$ symbol indicates the genotype of the male parent, while $q$ indicates the genotype of the female parent

were assigned to genomic features using featureCounts (Galaxy version 1.6.2) (Liao et al. 2014). Differential expression was analyzed using DESeq2 default parameters (Galaxy Version 2.11.40.6 + galaxy1) (Love et al. 2014). Differentially expressed genes (DEGs) were defined by an absolute $\log \mathrm{FC}>2$ and an FDR $<0.05$. Gene ontology (GO) enrichment analysis has been performed on PANTHER (Mi et al. 2019) and visualized using REVIGO (Supek et al. 2011). All plots have been generated using R-studio (www.rstudio. com). Raw data are deposited on the European Nucleotide Archive under reference PRJEB46544 (http://www.ebi.ac. uk/ena/data/view/PRJEB46544).

\section{Results}

\section{MET3 is expressed biallelically with a paternal bias in the endosperm}

Our previous analysis has shown that MET3 is expressed in the endosperm of developing Arabidopsis seeds (Jullien et al. 2012). However, the detail and exclusivity of its expression pattern remain to be investigated. To get a better characterization of MET3 expression pattern, we performed a qPCR of MET3 transcript in different tissue types of wild-type Col-0 (Fig. 1a). Our result shows that MET3 is principally expressed in siliques and its expression peaks at 5 Days After Pollination (DAP). These results could be confirmed using publicly available transcriptome datasets (Fig. S1a) (Klepikova et al. 2016). To gain a better spatial and temporal characterization of MET3 expression, we generated two new transcriptional MET3 reporter constructs, encompassing $2 \mathrm{~kb}$ of the MET3 promoter driving either H2B-tdTomato or H2B-GUS (Fig. S2a). The analysis of the pMET3:H2B-GUS in different plant tissues confirmed the specificity of MET3 expression to the seed endosperm (Fig. S1b-d). To characterize in detail the temporal expression of MET3 in the endosperm, we performed confocal microscopy on the pMET3:H2B-tdTomato lines at different stages of seed development. We could detect pMET3:H2B-tdTomato expression from as early as the four nuclei stage endosperm (Fig. 1b). pMET3:H2B-tdTomato remains express throughout the endosperm (Fig. 1c) until the globular stage of embryo development where its expression starts to be higher in the chalazal endosperm and chalazal cyst (Fig. 1d). At later stages, pMET3:H2BtdTomato expression is limited to the chalazal endosperm and chalazal cyst (Fig. 1e). From 7 DAP, pMET3:H2BtdTomato expression can no longer be detected. A similar expression pattern was observed with $p M E T 3: H 2 B-G U S$ (Fig. S2b) and pMET3:H2B-RFP (Fig. S2c) as well as online transcriptomic data (Fig. S2d) (Belmonte et al. 2013). MET3 protein expression and localization could not be determined as we, so far, failed in expressing a fluorescently tagged MET3 protein in Arabidopsis (LT personal communication).

Such endosperm expression pattern is common in imprinted genes, like FWA, FIS2, MEA or PHE1 (Kinoshita et al. 1999, 2004; Köhler et al. 2005; Jullien et al. 2006). In order to examine if MET3 is biallelically or mono-allelically expressed, we performed allele-specific RT-PCR. We are making use of a Short Nucleotide Polymorphism (SNP) consisting of a substitution from a $\mathrm{C}$ to a T within MET3 9th exon in the Gr-1 ecotype which is abolishing a XbaI restriction site present in Col-0. We did reciprocal crosses using Col-0 and Gr- 1 ecotypes and analyzed MET3 parental expression at 5 DAP following XbaI digestion (Fig. 2a). We could observe, for both reciprocal crosses, bands corresponding to MET3 transcript from Col-0 (505 and $329 \mathrm{bp}$ ) and from Gr-1 (834 bp) with a bias toward the paternal allele. This result shows that MET3 is expressed from both maternal and paternal allele but displays a paternal bias of expression. MET3 paternally biased expression was also observed using the pMET3:H2B-RFP transgene at 1 DAP (Fig. 2b). Taking together our results show that $M E T 3$ expression is biallelic with a paternal bias and confined to the endosperm. MET3 expression, initially throughout the endosperm, becomes restricted to the chalazal pole at later stages. 

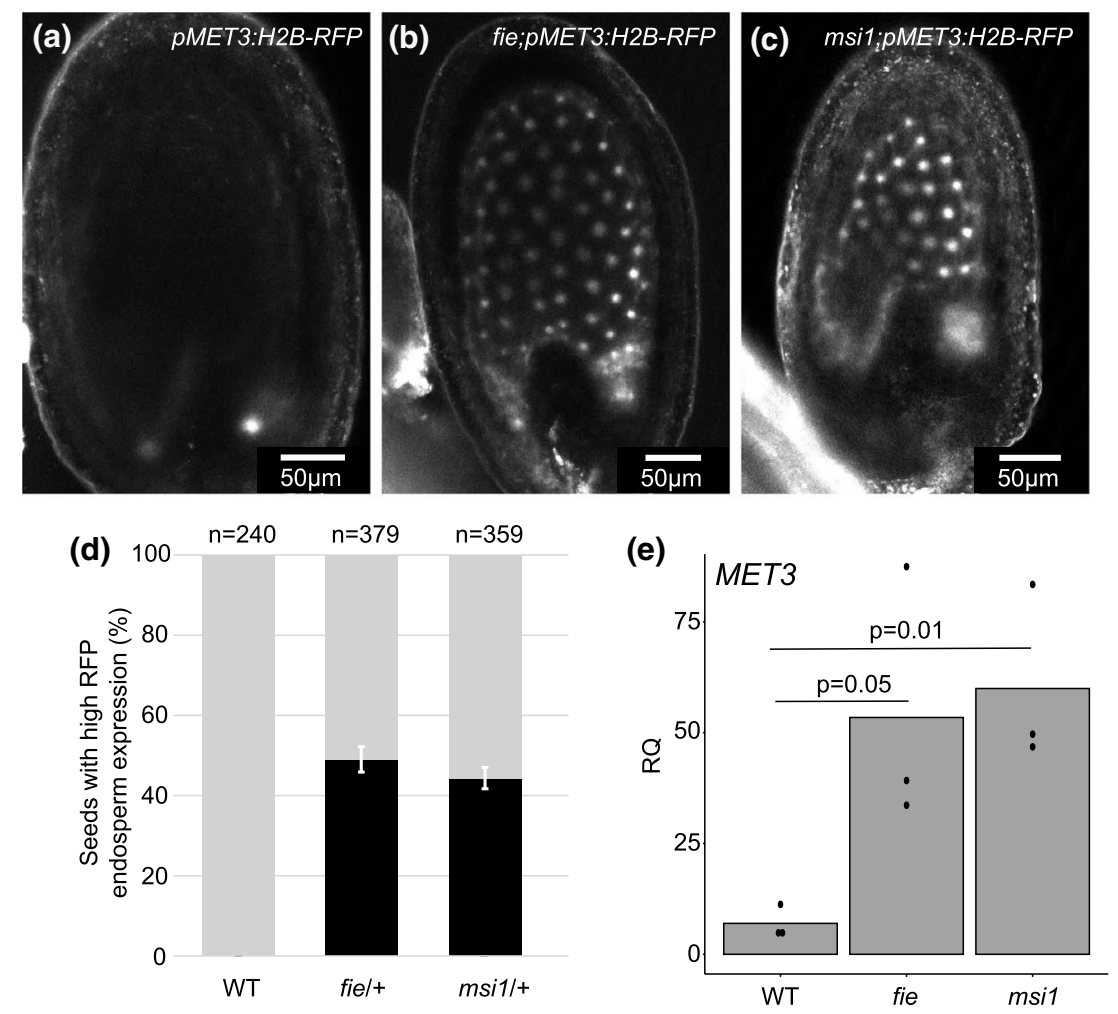

(f)

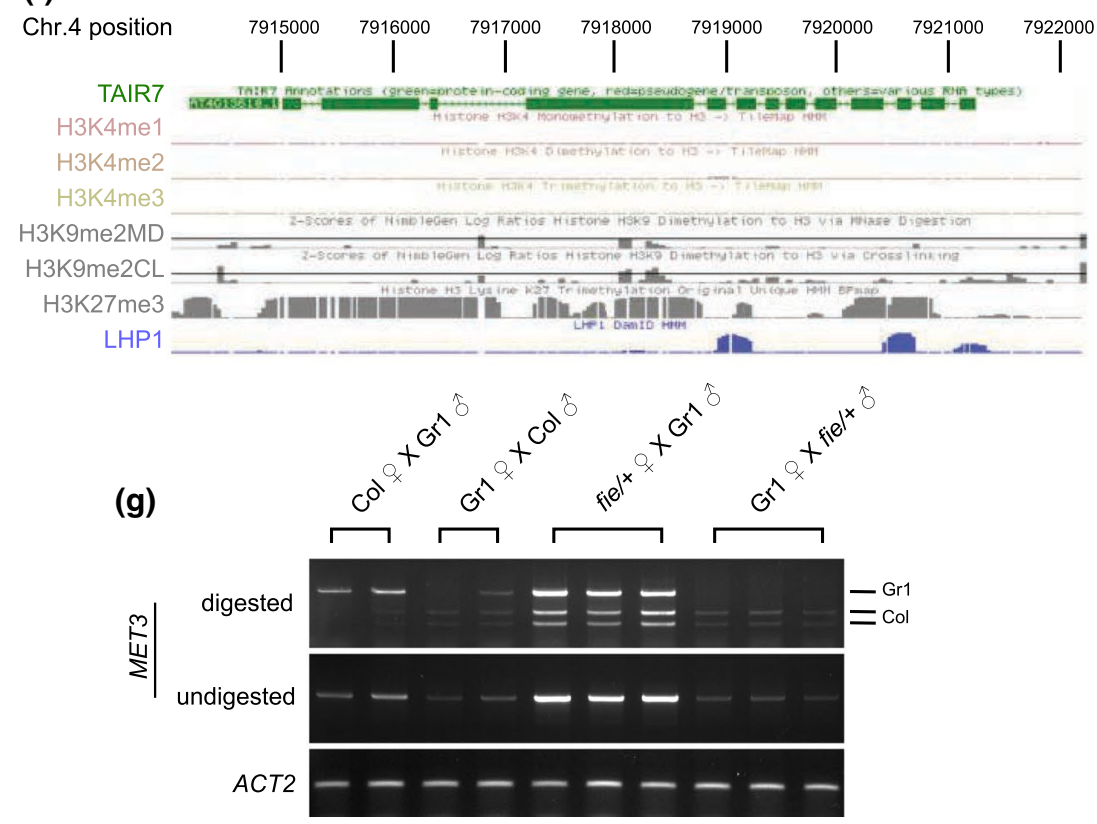

Fig. 3 MET3 expression is controlled by FIE and MSI1 Polycomb proteins a-c Confocal images representing the expression of pMET3:H2B-RFP construct in Arabidopsis wildtype a, fie b and msil c selfed seeds at 5 days after pollination (DAP). Scale bars represent $50 \mu \mathrm{m}$. d Proportion of seeds with high RFP signal in the endosperm at 5DAP in wild-type, fie and msil mutants. Grey bars represent the seeds with a restricted RFP expression to the chalazal endosperm (as illustrated in (a)). Black bars represent the seeds with ectopic expression of pMET3:H2B-RFP throughout the endosperm (as illustrated in (b-c)). e MET3 expression measured by RT-qPCR in
10 DAP selected seeds of wild-type, fie and msi1. The histogram displays the mean, and each dot represents a biological replicate. ACT2 is used as a normalizer. f Snapshot showing that the MET3 locus contains H3K27me3 and LHP1 but no H3K4me1, H3K4me2, H3K4me3 and $\mathrm{H} 3 \mathrm{~K} 9 \mathrm{me} 2$ in seedlings. Data from http://epigenomics.mcdb. ucla.edu/H3K27m3/ (Zhang et al. 2007). g Allele specific RT-PCR of MET3 parental expression in 5DAP silique samples of reciprocal crosses between wild-types and fie/+mutants (Col-0 background). The XbaI restriction enzyme digests the Col-0 MET3 transcript but not the Gr-1 transcript. ACT2 is used as loading control 


\section{MET3 expression is regulated by Polycomb group proteins}

Beyond imprinted genes, MET3 expression pattern is reminiscent of the expression of genes controlled by the endosperm FIS Polycomb group complex $(\mathrm{PcG})$ composed of FIE, MSI1, FIS2 and MEA (Guitton et al. 2004). To investigate if MET3 could be regulated by $\mathrm{PcG}$, we introgressed a MET3 transcriptional reporter into fie and msil mutant background (Fig. 3a-d). We could observe increased PMET3:H2B-RFP reporter expression in $49 \%$ of the seeds $(\mathrm{n}=379)$ in fie/ + mutant and $44 \%(n=359)$ in msil/+ mutant background characteristic of the maternal gametophytic effect of those mutations (Fig. 3d). In msi 1 and fie mutant seeds, pMET3:H2B-RFP expression is higher and observed throughout the endosperm (Fig. 3b-c), whereas at the same developmental stage the expression of pMET3:H2B-RFP is already restricted to the chalazal pole in wild-type seeds (Fig. 3a). Interestingly, this regulation seems restricted to the endosperm as no ectopic expression of pMET3:H2B-RFP was observed in the central cell, egg cell and sperm cells before fertilization (Fig. S3a-b). In order to confirm that the regulation of MET3 by MSII and FIE was not only restricted to our transgene, we performed a RT-qPCR of MET3 expression in wild-type and mutant selected seeds at 10 DAP (Fig. 3e). We could observe a clear upregulation of MET3 expression in both fie and msil seeds compare to wild-type seeds (t-test p-value of 0.0543 and 0.0114 , respectively). Using publicly available data, we could see that the upregulation of MET3 is also observed in other PcG mutants (Fig. S3c-d) such as the non FIS-PcG mutant siliques of $c l f$ (Fig. S3c) (Liu et al. 2016) and in the FIS-PcG mutant seeds of fis2 (Fig. S3d) (Weinhofer et al. 2010).

Subsequently, we wanted to know if the effect of the PcG complex was direct or indirect. PcG complexes are known to repress gene expression by tri-methylating the Lysine 27 of the Histone H3 tail (H3K27me3) inducing a closed chromatin state at the targeted loci. We, therefore, analyzed available H3K27me3 genome-wide Chromatin Immunoprecipitation (ChIP) data. We could see that the MET3 locus is covered by H3K27me3 in Arabidopsis seedling samples (Fig. 3f) (Zhang et al. 2007). Additionally, using the RepMap2020 tool (Chèneby et al. 2020), we could observe $\mathrm{H} 3 \mathrm{~K} 27 \mathrm{me} 3$ on the MET3 locus in several independent ChIP experiments including some performed on endosperm tissue (Fig. S3e). Interestingly, using publicly available allele specific endosperm H3K27me3 ChIP data (Moreno-Romero et al. 2016), we could observe that the maternal allele of MET3 appears to contain more $\mathrm{H} 3 \mathrm{~K} 27 \mathrm{me} 3$ than the paternal allele in reciprocal crosses using Col-0 and Ler (Fig S3f). To test if H3K27me3 mark is responsible for the silencing of the MET3 maternal allele, we performed an allele specific RT-PCR in reciprocal crosses using fie/+mutants (Fig. 3g). In crosses using fie/+as mother, we could observe biallelic expression of MET3 showing that the silencing of MET3 maternal allele rely on maternal FIE activity. It is also important to note that the paternal allele of MET3 is also repressed by maternal FIE (Fig. 3g). We conclude that MET3 expression and imprinting is directly regulated by $\mathrm{PcG}$ complex induced H3K27me3 in the endosperm.

\section{MET3 does not affect seed development}

Considering that MET3 is specifically expressed in the developing endosperm during seed development, we then ask if MET3 function influences seed and/or endosperm development. We characterized two mutant alleles from the GABI collection: met3-3 (GABI_404F04) and met3-4 (GABI_659H03) (Fig. S4a). The mutations are located on the 10th and the 2nd exon, respectively, and are expected to abolish MET3 function. To confirm the downregulation of MET3 in the mutants, we performed RT-qPCR. We could observe that MET3 is downregulated in both mutant alleles (Fig. S4b). To investigate if the met3 mutation could result in seed lethality we first investigated the presence of aborted seeds at the green seed stage ( 12DAP). We could not see any significant seed abortion in both met3-3 and met3-4 alleles (Fig. S5b). To further confirm the absence of defects, we analyzed the transmission rate of the met 3 mutations in $m e t 3-3 /+$ and met3-4/+ selfed progeny. We could not observe any segregation distortion from the Mendelian ratio (Fig. S5a). Mutations affecting the main Arabidopsis DNA methyltransferase $M E T 1$ display variation in seed size in reciprocal crossing with wild-type (Xiao et al. 2006). We, therefore, investigated if met 3 mutants would display a seed size phenotype in self and reciprocal crosses to wild-type. We could not see any significant variation in seed size using both met3 alleles (Fig. S5c-d). We conclude that MET3 mutation does not severely impair seed development. Additionally, met 1 mutant are known to display increased developmental defect through generation (Mathieu et al. 2007). To assess potential transgenerational effect of the met 3 mutation we have maintained met 3 homozygotes mutants for five generations. However, we could not observe such increased developmental phenotype with met 3 mutants after five generation of inbreeding $\left(m e t 3^{G 5}\right)$ (Fig. S5e-g). The $m e t 3^{G 5}$ did not show difference when compared to wild-type either in term of rosette size (Fig. S5f) nor flowering time (Fig. S5g). We conclude that MET3 mutations do not severely impair seed development and do not accumulate transgenerational developmental defects.

As shown above, MET3 expression is regulated by $\mathrm{PcG}$ complex in the endosperm, we then analyzed if MET3 mutation could influence the PcG fie mutant phenotype. To answer this question, we generated fiel $+;$ met $3-3$ and 

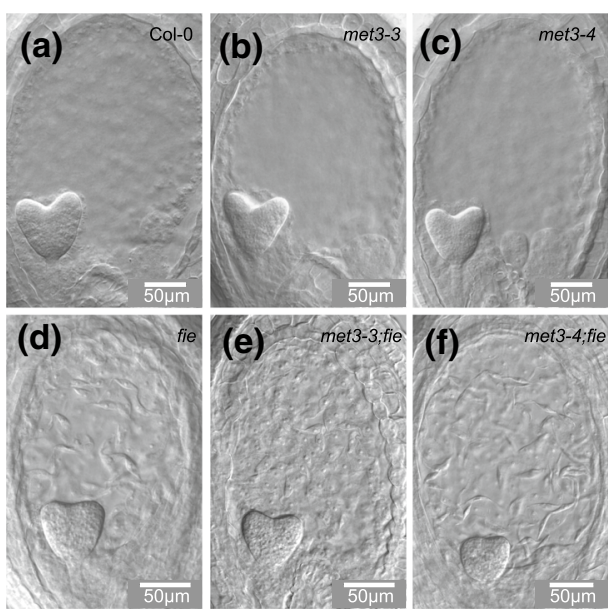

Fig. 4 MET3 does not influence fie phenotype (a-f) Seed developmental phenotype observed after chloral hydrate clearing by Difference Interference Contrast (DIC) of wild-type a, met3-3 b, met3-4 c, fie/ + d, met3-3; fie/ +e and met3-4;fie/ + f at 6 days after synchronization (DAS). Scale bars represent $50 \mu \mathrm{m}$. g-h Quantification of the embryo developmental stages $\mathbf{g}$ and endosperm cellularization $\mathbf{h}$ in

fiel + ;met3-4 double mutants and analyzed their seed development phenotype using DIC (Fig. 4a-h). In order to minimize the stress to the plant due to handling during emasculation and crossing, we used "synchronized seeds." In practice, we remove the open flowers of the day, and we wait an $\mathrm{x}$ number of days before collecting two siliques above our cutting. This is allowing us to have age synchronized siliques without the physical disturbance of emasculation/pollination and is, therefore, closer to normal growth and fertilization. We are using the term Day After Synchronization (DAS). A side-by-side comparison between DAS and DAP can be found in Fig. S6a-c. To our experience, 6 DAS is corresponding to around 4-5DAP. At 6 DAS, we could not see any delay in embryo development and endosperm cellularization comparing Col-0 to met3-3 and met3-4 mutants (Fig. 4a-c, g-h). In fiel+, we could clearly see the delayed endosperm cellularization characteristic of FIS-PcG mutants (Fig. 4d-f and g-h) (56\% $n=407$ ) (Ohad et al. 1999; Sørensen et al. 2001). In the double mutants, we could not see variations in the quantification of the fie/ + phenotype for both embryo development (Fig. 4g) and endosperm cellularization (Fig. 4h). An illustration of the endosperm cellularization categories can be found in Fig. S7a. An additional well-known phenotype of FIS-PcG mutants is the development of autonomous seed in the absence of fertilization. We could not see any variation of the autonomous seed development phenotype in met3/-;fie/+double mutant compared to fie/+ (Fig. S7bc). We conclude that MET3 mutations do not influence fie mutant seed phenotype.

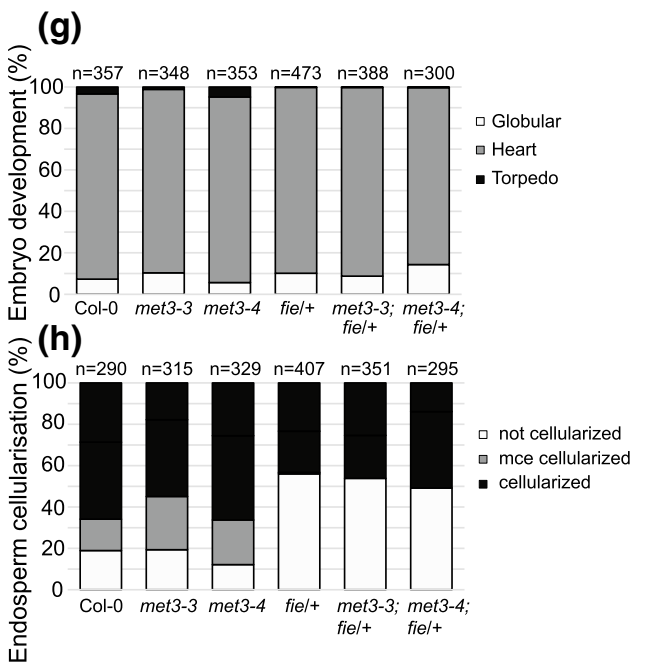

wild-type, met3-3, met3-4, fie/+, met3-3;fie/+ and met3-4;fie/ + at 6 DAS. mce $=$ micropylar endosperm. Images illustrating the endosperm scoring categories can be found in Fig. S7a. An analysis of seed autonomous development in fie/+, met3-3; fie/+and met34;fie/ + can be found in Fig S7b-c

\section{Effect of MET3 mutation on the seed transcriptome}

In order to investigate the potential effect of met 3 mutation on the seed transcriptome, we performed a mRNA deep sequencing experiment of 3 DAP (Fig. S8) and 10 DAS (Fig. 5a) seeds (i.e., seeds attached to the septum). We then compared the seed transcriptome of met 3 seeds to wild-type seeds. At 3 DAP, we could only detect one differentially expressed gene: ESM1 (AT3G14210). ESM1 was up-regulated in both met3-3 and met3-4 mutant seeds (Fig. S8a-b). At 10 DAS, we could also only see very minor changes to the seed transcriptome (Fig. 5a). We could identify only 16 differentially expressed genes with an absolute $\log \mathrm{FC}>2$ and FDR $<0.05$. These results show that reminiscent of the absence of seed phenotype in met 3 mutants, the seed transcriptome is also mostly unaffected by the met 3 mutation. As MET3 expression is regulated by the FIS-PcG complex in the endosperm, we then ask if MET3 mutation could influence the fie transcriptome. We, therefore, sequenced the transcriptome of 10 DAS seeds where we selected for fie mutant seeds under the dissecting microscope (white seeds). We compared the transcriptome of fie seeds to the transcriptome of fie;met3-3 double mutant seeds. We could observe 87 differentially expressed genes (DEG) with an absolute $\log F C>2$ and FDR $<0.05$ (Fig. 5b). A Goterm enrichment analysis revealed that these genes are enriched for genes involved in pectin metabolism (GO:0,045,490, FDR $=4.46 \mathrm{E}-10$; GO:0,045,488, FDR $=6.59 \mathrm{E}-09$ ) and cell-wall related processes (GO:0,042,545, FDR = 8.97E-09; GO:0,071,555, 

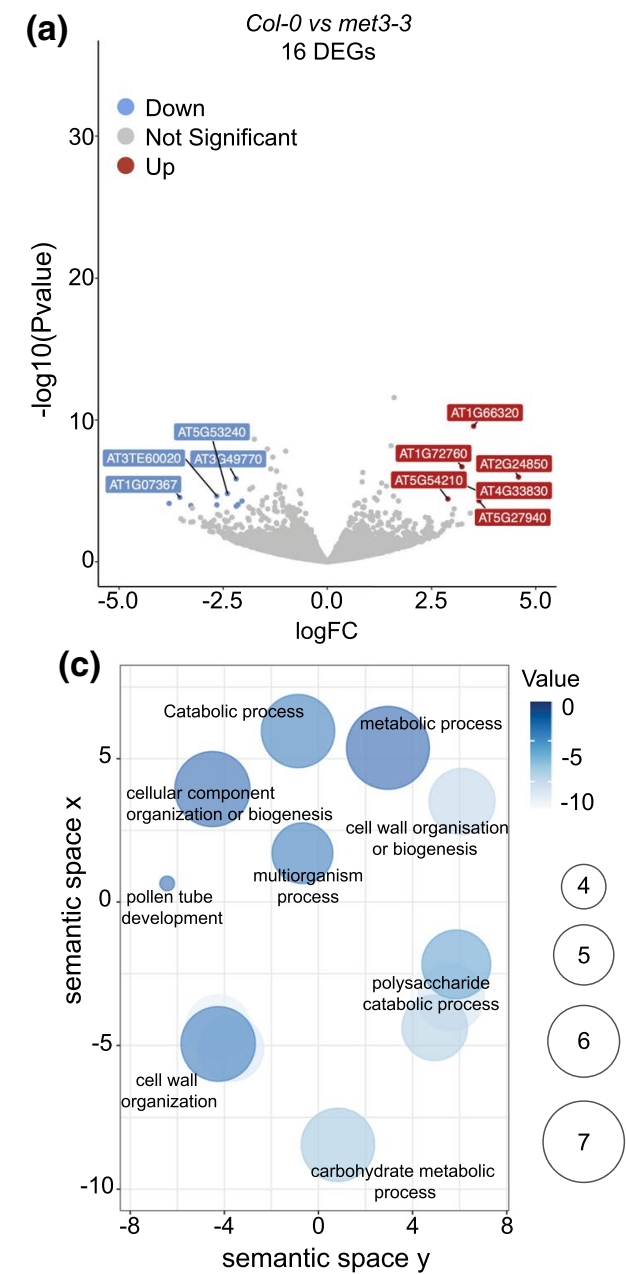

Fig. 5 Transcriptome of met3 and met3;fie mutant seeds a-b Volcano plot depicting differentially expressed genes (DEGs) comparing Col-0 to met3-3 a and fie to met3-3;fie in 10 DAS seeds b. Red dots represent up-regulated DEGs and blue dots represent down-regulated

FDR $=4.87 \mathrm{E}-10$, GO:0,071,554, FDR $=5.67 \mathrm{E}-08)($ Fig. $5 \mathrm{c}$ and Table S2). We then analyzed if among these 87 DEGs some are also modified in fie mutant compared to wildtype. We could find that a large proportion of either met3 DEGs (Col vs met3,11/16) and met3;fie DEGs (fie versus met3;fie, 44/87) are miss-regulated in fie mutant seeds (Fig. 5d). We conclude that the met3 mutation does not drastically change the seed transcriptome, but a set of genes seem miss-regulated by both met 3 and fie mutants.

\section{Discussion}

Our study highlights an additional connection between DNA Methylation pathways and Polycomb group H3K27 tri-methylation in the seed endosperm via the regulation of MET3 by the FIS-PcG complex. MET3 is specifically expressed

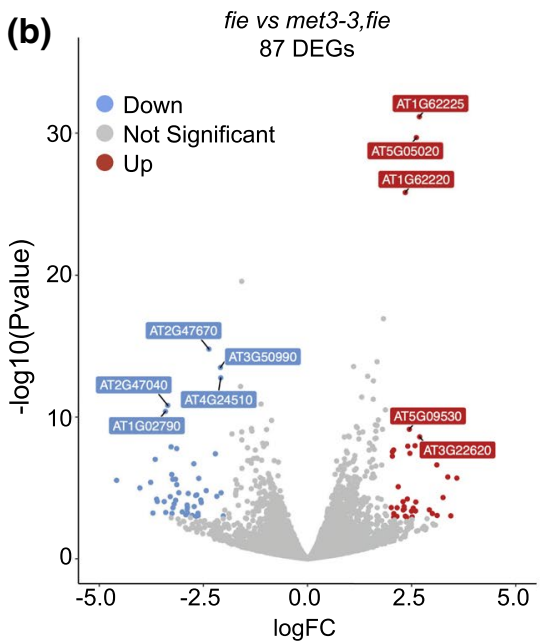

(d)

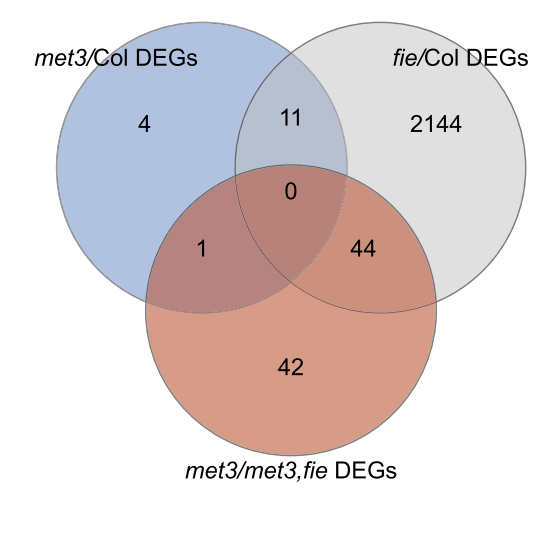

DEGs. The top 10 DEGs are annotated. We use a threshold of [absolute $\log \mathrm{FC}>2$, FDR $<0.05]$. c GO term analysis for the 87 DEGs obtained with the fie vs met3-3;fie contrast. d Venn diagram showing the overlapping DEGs between the different contrasts

in the endosperm, and its expression becomes restricted to the chalazal pole at later stages of endosperm development. The analysis of the transcriptome of met 3 mutant seeds did not reveal a clear function of MET3 in regulating gene expression in the endosperm. Such regulation could have been masked by a dilution effect as we analyzed seed transcriptome rather than endosperm transcriptome. MET3 role in wild-type endosperm as a functional DNA methyltransferase remains to be investigated. Despite being hypomethylated, the endosperm DNA is still methylated (Hsieh et al. 2009). Which DNA methyltransferases participate is currently unknown but MET3 is a good candidate. Future studies investigating the DNA methylation level in a met 3 endosperm will allow to test this hypothesis.

In our study, using two independent insertion lines, met33 and met3-4, we could not observe any major seed developmental phenotype. Both mutants show a significant decrease 
in MET3 expression by qPCR suggesting they both represent knockout mutants. It was previously documented that a mutation affecting MET3 named mee57 displayed a strong seed developmental phenotype (Pagnussat et al. 2005). The mee 57 mutation shows an early maternal embryo and endosperm arrest. The discrepancy between our lines and the previously published mee 57 could have several causes: the mutagenesis method used (T-DNA versus transposition), the presence of additional genetic modifications, or a difference between the two ecotypes used, Columbia- 0 in our case and Landsberg for mee57. If the latter is true, MET3 function could vary between different Arabidopsis accessions.

In this work, we show that repression of MET3 expression at later stages of endosperm development is linked to the direct action of the FIS-PcG complex on the MET3 locus. MET3 is over-expressed in PcG mutant seeds, such as fie and msil mutant seeds. Interestingly, it was previously shown that fie mutant endosperm display higher CG methylation compared to wild-type endosperm and lower $\mathrm{CHG}$ and $\mathrm{CHH}$ (Ibarra et al. 2012). This increased CG methylation in fie mutant seems to be restricted to the endosperm as it is not observed in fie seedling or fie embryo methylome (Bouyer et al. 2017). We propose that higher expression of MET3 in the fie endosperm could be the cause for the increase in CG methylation specifically in the endosperm. Indeed, in our study, we could not observe any change in MET3 tissue expression pattern in PcG mutants but the increased expression is still restricted to the endosperm. In further studies, the Investigation of the endosperm methylome in met 3 and met3;fie mutants will allow to test this hypothesis.

As we previously mentioned, MET3 function could vary in between wild Arabidopsis accessions. Similarly, to CMT1, several SNPs can be found at the MET3 locus in-between different ecotypes suggesting that MET3 might not be fully functional in all of them. Additionally, looking closer at the MET3 locus, we could detect the presence of a Class 2 DNA transposon (AT4TE34810) situated in the third intron of the MET3 gene. In addition to PcG regulation, MET3 expression and possibly function could be influenced by the presence or regulation of AT4TE34810 like it is the case for the $C M T 1$ transcript (Yadav et al. 2018). The study of MET3 function and imprint in different accession could reveal more about its function in natural growth conditions. One hint of MET3 potential function in Columbia-0 came from the study of methylome stability across several generations (Becker et al. 2011). In this study, methylome stability was investigated in several Col-0 selfed lineages (over 30 generations). The line accumulating the most methylation polymorphisms had concomitantly acquired a SNP in the MET3 gene. It was therefore suggested that MET3 could be the cause of such methylome instability (Becker et al. 2011; Schmitz and Ecker 2012). Analyzing the methylome of selfed met 3 mutants after 30 generations would allow to test this hypothesis. If true and considering the endosperm specificity of MET3 expression, it would indicate that the endosperm methylome influences the embryonic methylome, a hypothesis that has stimulated a lot of interest over the last 10 years but remains to be demonstrated.

Supplementary Information The online version contains supplementary material available at https://doi.org/10.1007/s00497-021-00436-x.

Acknowledgements We would like to thank the following people for their help: Jasmine Sekulovski for support concerning plant growth. We thank Mathieu Ingouff for critical reading of the manuscript.

Author contribution statement PEJ conceived the study. LT performed the experiments. DMVB contributed Fig S3g. LT and PEJ analyzed the data. PEJ wrote the manuscript with the help of LT.

Funding PEJ, DBMV and LT are supported by an SNF professorship grant (no.163946) attributed to PEJ. schweizerischer nationalfonds zur förderung der wissenschaftlichen forschung, 163946,pauline emilie jullien

\section{Declarations}

Conflict of interest Authors state that there is no conflict of interest.

Open Access This article is licensed under a Creative Commons Attribution 4.0 International License, which permits use, sharing, adaptation, distribution and reproduction in any medium or format, as long as you give appropriate credit to the original author(s) and the source, provide a link to the Creative Commons licence, and indicate if changes were made. The images or other third party material in this article are included in the article's Creative Commons licence, unless indicated otherwise in a credit line to the material. If material is not included in the article's Creative Commons licence and your intended use is not permitted by statutory regulation or exceeds the permitted use, you will need to obtain permission directly from the copyright holder. To view a copy of this licence, visit http://creativecommons.org/licenses/by/4.0/.

\section{References}

Afgan E, Baker D, Batut B et al (2018) The Galaxy platform for accessible, reproducible and collaborative biomedical analyses: 2018 update. Nucleic Acids Res 46:W537-W544. https://doi.org/10. 1093/nar/gky379

Andrews S (2010) FastQC: a quality control tool for high throughput sequence data.

Batista RA, Köhler C (2020) Genomic imprinting in plants-revisiting existing models. Genes Dev 34:24-36. https://doi.org/10.1101/ gad.332924.119

Becker C, Hagmann J, Müller J et al (2011) Spontaneous epigenetic variation in the Arabidopsis thaliana methylome. Nature 480:245249. https://doi.org/10.1038/nature10555

Belmonte MF, Kirkbride RC, Stone SL et al (2013) Comprehensive developmental profiles of gene activity in regions and subregions of the Arabidopsis seed. Proc Natl Acad Sci 110:E435-E444. https://doi.org/10.1073/pnas.1222061110 
Bensmihen S, To A, Lambert G et al (2004) Analysis of an activated ABI5 allele using a new selection method for transgenic Arabidopsis seeds. FEBS Lett 561:127-131. https://doi.org/10.1016/ S0014-5793(04)00148-6

Berger F (2003) Endosperm: the crossroad of seed development. Curr Opin Plant Biol 6:42-50. https://doi.org/10.1016/S136952660 2000043

Boisnard-Lorig C, Colon-Carmona A, Bauch M et al (2001) Dynamic analyses of the expression of the HISTONE::YFP fusion protein in arabidopsis show that syncytial endosperm is divided in mitotic domains. Plant Cell 13:495-509

Bolger AM, Lohse M, Usadel B (2014) Trimmomatic: a flexible trimmer for Illumina sequence data. Bioinformatics 30:2114-2120. https://doi.org/10.1093/bioinformatics/btu170

Bouyer D, Kramdi A, Kassam M et al (2017) DNA methylation dynamics during early plant life. Genome Biol 18:179. https://doi.org/ 10.1186/s13059-017-1313-0

Bouyer D, Roudier F, Heese M et al (2011) Polycomb repressive complex 2 controls the embryo-to-seedling phase transition. PLoS Genet. https://doi.org/10.1371/journal.pgen.1002014

Brown RC, Lemmon BE, Nguyen H (2003) Events during the first four rounds of mitosis establish three developmental domains in the syncytial endosperm of Arabidopsis thaliana. Protoplasma 222:167-174. https://doi.org/10.1007/s00709-003-0010-x

Brown RC, Lemmon BE, Nguyen H, Olsen OA (1999) Development of endosperm in Arabidopsis thaliana. Sex Plant Reprod 12:32-42. https://doi.org/10.1007/s004970050169

Cao X, Jacobsen SE (2002) Locus-specific control of asymmetric and CpNpG methylation by the DRM and CMT3 methyltransferase genes. Proc Natl Acad Sci U S A 99:16491-16498. https://doi. org/10.1073/pnas.162371599

Chaudhury AM, Ming L, Miller C et al (1997) Fertilization-independent seed development in Arabidopsis thaliana. Proc Natl Acad Sci U S A 94:4223-8

Choi Y, Gehring M, Johnson L et al (2002) DEMETER, a DNA glycosylase domain protein, is required for endosperm gene imprinting and seed viability in arabidopsis. Cell 110:33-42

Chèneby J, Ménétrier Z, Mestdagh M et al (2020) ReMap 2020: a database of regulatory regions from an integrative analysis of Human and Arabidopsis DNA-binding sequencing experiments. Nucleic Acids Res 48:D180-D188. https://doi.org/10.1093/nar/gkz945

Clough SJ, Bent AF (1998) Floral dip: a simplified method for Agrobacterium-mediated transformation of Arabidopsis thaliana. Plant J 16:735-743. https://doi.org/10.1046/j.1365-313X.1998.00343.x

Costa LM, Gutièrrez-Marcos JF, Dickinson HG (2004) More than a yolk: the short life and complex times of the plant endosperm. Trends Plant Sci 9:507-514. https://doi.org/10.1016/j.tplants. 2004.08.007

Gehring M (2013) Genomic imprinting: insights from plants. Annu Rev Genet 47:187-208. https://doi.org/10.1146/annur ev-genet-110711-155527

Gehring M, Bubb KL, Henikoff S (2009) Extensive demethylation of repetitive elements during seed development underlies gene imprinting. Science 324:1447-51. https://doi.org/10.1126/scien ce. 1171609

Gehring M, Huh JH, Hsieh TF et al (2006) DEMETER DNA glycosylase establishes MEDEA polycomb gene self-imprinting by allele-specific demethylation. Cell 124:495-506. https://doi.org/ 10.1016/j.cell.2005.12.034

Guitton A, Page DR, Chambrier P et al (2004) Identification of new members of Fertilisation Independent Seed Polycomb Group pathway involved in the control of seed development in Arabidopsis thaliana. Development. https://doi.org/10.1242/dev.01168

Henikoff S, Comai L (1998) A DNA Methyltransferase Homolog With a Chromodomain Exists in Multiple Polymorphic Forms in Arabidopsis. Genetics 149:307-318
Hsieh TT-F, Ibarra CA, Silva P et al (2009) Genome-wide demethylation of Arabidopsis endosperm. Science 324:1451-1454. https:// doi.org/10.1126/science.1172417

Ibarra CA, Feng X, Schoft VK et al (2012) Active DNA Demethylation in Plant Companion Cells Reinforces Transposon Methylation in Gametes. Science 337:1360-1364. https://doi.org/10.1126/scien ce. 1224839

Jullien PE, Berger F (2009) Gamete-specific epigenetic mechanisms shape genomic imprinting. Curr Opin Plant Biol 12:637-642. https://doi.org/10.1016/j.pbi.2009.07.004

Jullien PE, Kinoshita T, Ohad N, Berger F (2006) Maintenance of DNA Methylation during the Arabidopsis Life Cycle Is Essential for Parental Imprinting. Plant Cell 18:1360-1372. https://doi.org/ 10.1105/tpc.106.041178.1

Jullien PE, Susaki D, Yelagandula R et al (2012) DNA methylation dynamics during sexual reproduction in Arabidopsis thaliana. Curr Biol 22:1825-1830. https://doi.org/10.1016/j.cub.2012.07. 061

Karimi M, De Meyer B, Hilson P (2005) Modular cloning in plant cells. Trends Plant Sci 10:103-105. https://doi.org/10.1016/j.tplan ts.2005.01.008

Kim D, Langmead B, Salzberg SL (2015) HISAT: A fast spliced aligner with low memory requirements. Nat Methods 12:357-360. https:// doi.org/10.1038/nmeth.3317

Kinoshita T, Yadegari R, Harada JJ et al (1999) Imprinting of the MEDEA polycomb gene in the Arabidopsis endosperm. Plant Cell 11:1945-1952

Kinoshita T, Miura A, Choi Y, et al (2004) One-Way Control of FWA Imprinting in Arabidopsis Endosperm by DNA Methylation. Science (80- ) 303:521-523. https://doi.org/10.1126/science.1089835

Kiyosue T, Ohad N, Yadegari R et al (1999) Control of fertilizationindependent endosperm development by the MEDEA polycomb gene in Arabidopsis. Proc Natl Acad Sci 96:4186-4191. https:// doi.org/10.1073/pnas.96.7.4186

Klepikova AV, Kasianov AS, Gerasimov ES et al (2016) A high resolution map of the Arabidopsis thaliana developmental transcriptome based on RNA-seq profiling. Plant J 88:1058-1070. https://doi. org/10.1111/tpj.13312

Köhler C, Hennig L, Bouveret R et al (2003) Arabidopsis MSI1 is a component of the MEA/FIE Polycomb group complex and required for seed development. EMBO J 22:4804-4814. https:// doi.org/10.1093/emboj/cdg444

Köhler C, Page DR, Gagliardini V, Grossniklaus U (2005) The Arabidopsis thaliana MEDEA Polycomb group protein controls expression of PHERES1 by parental imprinting. Nat Genet 37:28-30. https://doi.org/10.1038/ng1495

Liao Y, Smyth GK, Shi W (2014) FeatureCounts: An efficient general purpose program for assigning sequence reads to genomic features. Bioinformatics 30:923-930. https://doi.org/10.1093/bioin formatics/btt656

Lindroth a M, Cao X, Jackson JP, et al (2001) Requirement of CHROMOMETHYLASE3 for maintenance of CpXpG methylation. Science 292:2077-2080. https://doi.org/10.1126/science.1059745

Liu J, Deng S, Wang H et al (2016) CURLY LEAF regulates gene sets coordinating seed size and lipid biosynthesis. Plant Physiol 171:424-436. https://doi.org/10.1104/pp.15.01335

Love MI, Huber W, Anders S (2014) Moderated estimation of fold change and dispersion for RNA-Seq data with DESeq2. Genome Biol 15:550. https://doi.org/10.1186/s13059-014-0550-8

Luo M, Bilodeau P, Dennis ES et al (2000) Expression and parentof-origin effects for FIS2, MEA, and FIE in the endosperm and embryo of developing Arabidopsis seeds. Proc Natl Acad Sci U S A. https://doi.org/10.1073/pnas.170292997

Luo M, Bilodeau P, Koltunow a, et al (1999) Genes controlling fertilization-independent seed development in Arabidopsis thaliana. Proc Natl Acad Sci U S A 96:296-301 
Mathieu O, Reinders J, Caikovski M et al (2007) Transgenerational stability of the Arabidopsis epigenome is coordinated by CG methylation. Cell 130:851-862. https://doi.org/10.1016/j.cell. 2007.07.007

Mi H, Muruganujan A, Huang X et al (2019) Protocol Update for largescale genome and gene function analysis with the PANTHER classification system (v.14.0). Nat Protoc 14:703-721. https://doi.org/ 10.1038/s41596-019-0128-8

Moreno-Romero J, Jiang H, Santos-González J, Köhler C (2016) Parental epigenetic asymmetry of PRC2-mediated histone modifications in the Arabidopsis endosperm. EMBO J 35:1298-1311. https:// doi.org/10.15252/embj.201593534

Ohad N, Yadegari R, Margossian L et al (1999) Mutations in FIE, a WD polycomb group gene, allow endosperm development without fertilization. Plant Cell 11:407-415. https://doi.org/10.1105/tpc. 11.3.407

Pagnussat GC, Yu HJ, Ngo QA et al (2005) Genetic and molecular identification of genes required for female gametophyte development and function in Arabidopsis. Development 132:603-614. https://doi.org/10.1242/dev.01595

Quadrana L, Silveira AB, Mayhew GF et al (2016) The Arabidopsis thaliana mobilome and its impact at the species level. Elife 5:1-25. https://doi.org/10.7554/eLife.15716

Salvador-Guirao R, Baldrich P, Weigel D et al (2018) The MicroRNA miR773 Is Involved in the Arabidopsis Immune Response to Fungal Pathogens. Mol Plant-Microbe Interact 31:249-259. https:// doi.org/10.1094/MPMI-05-17-0108-R

Schmitz RJ, Ecker JR (2012) Epigenetic and epigenomic variation in Arabidopsis thaliana. Trends Plant Sci 17:149-154. https://doi. org/10.1016/j.tplants.2012.01.001

Stroud H, Do T, Du J et al (2013) Non-CG methylation patterns shape the epigenetic landscape in Arabidopsis. Nat Struct Mol Biol 21:64-72. https://doi.org/10.1038/nsmb.2735
Supek F, Bošnjak M, Škunca N, Šmuc T (2011) Revigo summarizes and visualizes long lists of gene ontology terms. PLoS ONE. https://doi.org/10.1371/journal.pone.0021800

Sørensen MB, Chaudhury AM, Robert H et al (2001) Polycomb group genes control pattern formation in plant seed. Curr Biol 11:277-281

Weinhofer I, Hehenberger E, Roszak P et al (2010) H3K27me3 profiling of the endosperm implies exclusion of polycomb group protein targeting by DNA methylation. PLoS Genet 6:e1001152. https:// doi.org/10.1371/journal.pgen.1001152

Winter D, Vinegar B, Nahal H et al (2007) An "electronic fluorescent pictograph" Browser for exploring and analyzing large-scale biological data sets. PLoS ONE 2:1-12. https://doi.org/10.1371/journ al.pone. 0000718

Xiao W, Brown RC, Lemmon BE et al (2006) Regulation of seed size by hypomethylation of maternal and paternal genomes. Plant Physiol 142:1160-1168. https://doi.org/10.1104/pp.106.088849

Yadav NS, Khadka J, Domb K et al (2018) CMT3 and SUVH4/KYP silence the exonic Evelknievel retroelement to allow for reconstitution of CMT1 mRNA Epigenetics \& Chromatin 11:1-12. https://doi.org/10.1186/s13072-018-0240-y

Yadegari R, Kinoshita T, Lotan O et al (2000) Mutations in the FIE and MEA genes that encode interacting polycomb proteins cause parent-of-origin effects on seed development by distinct mechanisms. Plant Cell 12:2367-2382

Zhang X, Clarenz O, Cokus S et al (2007) Whole-genome analysis of histone $\mathrm{H} 3$ lysine 27 trimethylation in Arabidopsis. PLoS Biol 5:e129. https://doi.org/10.1371/journal.pbio.0050129

Publisher's Note Springer Nature remains neutral with regard to jurisdictional claims in published maps and institutional affiliations. 